\title{
THE MAGNETIZATION OF DENSE AGGREGATED DIPOLAR FLUIDS
}

\author{
Sándor Nagy \\ Institute of Mechatronics Engieering and Research, Gasparich M. u. 18/A, 8900, Zalaegerszeg, Hungary, \\ e-mail:sata123.sandor@gmail.com
}

\begin{abstract}
The magnetization for dipolar fluids is studied treated separately at low and high external magnetic field. Canonical ensemble Monte Carlo simulations have been performed in dipolar hard sphere fluid in order to test these theoretical results. New expressions are introduced at low and high external field and ultimately the synthesized formula is given. The main difference in the structure of dipolar liquids at different external field is the average orientation of the formed chains. In case of infinitesimal external magnetic field there is no a well-specified direction, while at high enough external field actually the chains are parallel to this field. The present theory yields good result in the intermediate region as well where the most conspicuous failures are provided by the former theories.
\end{abstract}

Keywords: dipolar fluids, magnetization, ferrofluids, Monte Carlo simulation

\section{INTRODUCTION}

\subsection{The effective field}

There are several relevant theories for the magnetization of dipolar fluids. These differ from each other mainly that in the expression of magnetization that

$$
M=\rho m L\left(\frac{m H_{e}}{k_{B} T}\right)
$$

the $H_{e}$ effective field is determined differently. Hereinafter the $m H / k_{B} T$ type argument in the $L$ Langevin function will be denoted by $\alpha$. The index of $\alpha$ will be the same as index of $H$ magnetic field. In (1) $M$ is the magnetization, $\rho$ is the number density, $m$ is the dipole moment, $k_{B}$ is the Boltzmann constant, and $T$ is the temperature. According to the Langevin theory [1] the effective magnetic field is equal to the external magnetic field:

$$
H_{e}=H_{0}
$$

i.e. it does not take into account the field strength from adjacent particles. In his forward theory, Weiss [2] has gave an implicit expression to $H_{e}$ :

$$
H_{e}=H_{0}+\frac{4 \pi}{3} \rho m L\left(\alpha_{e}\right)
$$


Here the effective field is equal to the sum of external field and additional field from the surrounding particles. This leads to the outcome, that the effective field is not equal to zero when the external field is zero, which is obviously incorrect. Although Pschenichnikov [3] has made a small change in the argument of Langevin function, but the divergence disappeared:

$$
H_{e}=H_{0}+\frac{4 \pi}{3} \rho m L\left(\alpha_{0}\right)
$$

Equation (4) describes well the magnetization at low density and dipole moment, although at low external field underestimates and at high external field overestimates the simulation data. Ivanov [4] completes the second term in (4) in order to that the results at low external magnetic field get closer to the simulations. This has more or less succeeded but in this case (5) even more overestimates the simulations at high external magnetic field:

$$
H_{e}=H_{0}+\frac{4 \pi}{3} \rho m L\left(\alpha_{0}\right)\left[1+\frac{4 \pi}{48} \frac{\partial\left(\rho m L\left(\alpha_{0}\right)\right)}{\partial H_{0}}\right]
$$

On Fig. 1 the curves of effective field according to some former theory are presented in function of external field at four different values of density and dipole moment. Furthermore the simulation values of effective field are marked also by the help of (1) calculated from the simulation values of magnetization. Now and hereafter on the figures the reduced units are used, defined by these expressions: $\rho^{*}=\rho \sigma^{3}$, $m^{*}=m / \sqrt{\sigma^{3} k_{B} T}, M^{*}=M \sqrt{\sigma^{3} / k_{B} T}, H^{*}=H \sqrt{\sigma^{3} / k_{B} T}$. (The $\sigma$ is the diameter of particles of monodisperse dipolar hard sphere system.) 

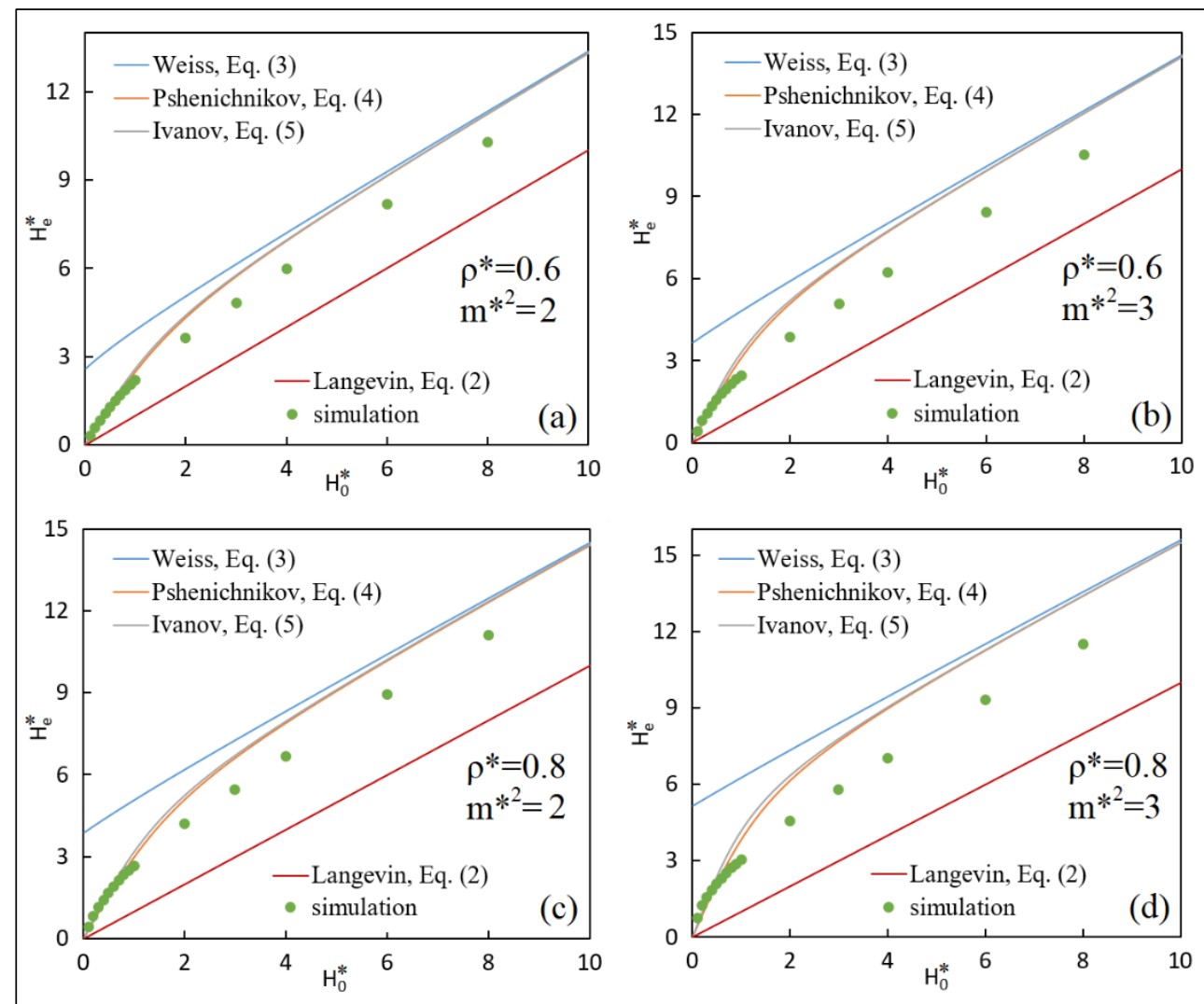

Figure 1. The values of effective magnetic field in function of external magnetic field in case of four former theories and simulation.

It is clearly shown on Fig. 1, that in all four cases there are significant difference between the simulation and theoretical data for both large and small external field. As the external field and thus the effective field increases, in the magnetization this difference disappears ever since the magnetization goes to saturated. All three relevant theories (Weiss, Pshenichnikov, Ivanov) as shown in Fig. 2 lead to the same value of magnetization, when $H_{0} \rightarrow \infty$. There are even more theories for the magnetization of dipolar fluids, which have approached the experimental and simulation values with more or less success [5-9]. 


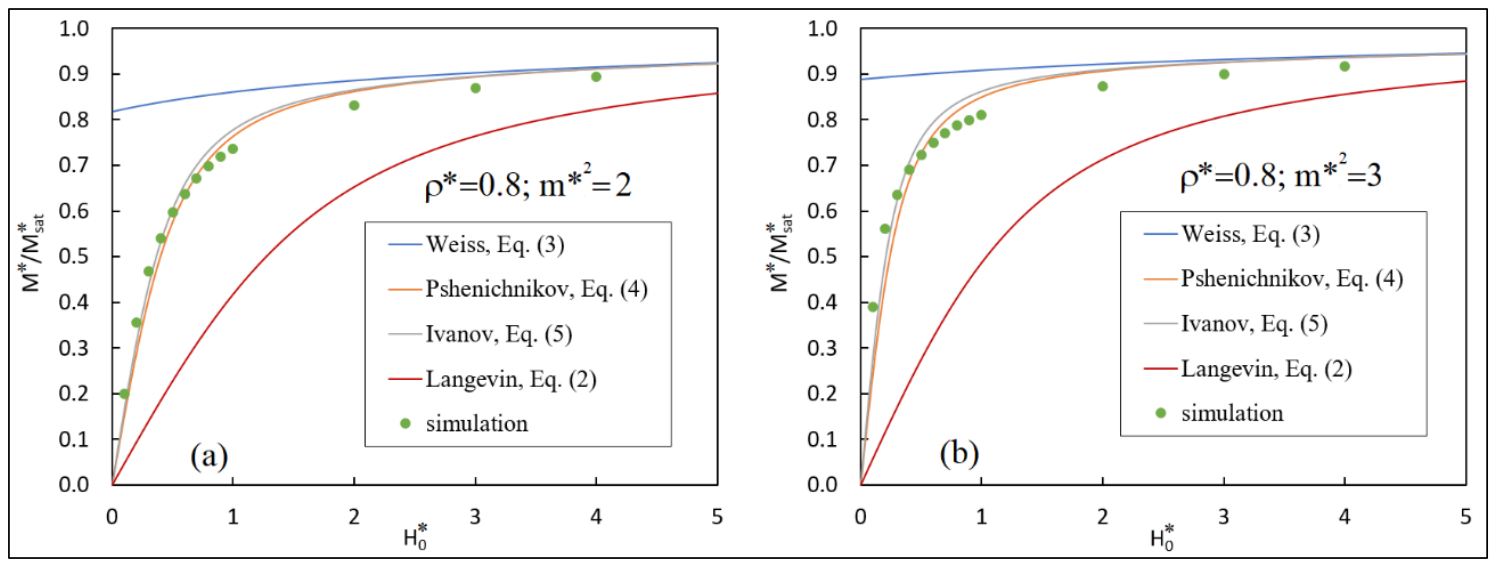

Figure 2. The values of magnetization curve in function of external magnetic field in case of four former theories and simulation.

\subsection{The closure of effective field at zero external field}

For the initial susceptibility of dense dipolar fluids recently Nagy [10] has given new theoretical results. The new method is based on that dipoles are chained already at zero external field. The chain and particle distribution can be calculated according to the geometric distribution. The chain distribution is

$$
g_{k}=q p^{k-1}
$$

where $p$ is the probability of chaining between two adjacent, parallel particles, are under the influence of local magnetic field, while $q=1-p$, and $k$ is the length of the chain. The particle distribution specifies the proportion of particles involved in $k$ length chains:

$$
h_{k}=q^{2} k p^{k-1}
$$

According to this theory the initial magnetic susceptibility can be calculated as follows:

$$
\chi=\frac{1+p}{1-p} \chi_{L}\left(1+\frac{4 \pi}{3} \chi_{L}\right)
$$

where $\chi_{L}=\rho m^{2} / 3 k_{B} T$ is Langevin susceptibility. For dipolar hard sphere system the values of $p$ to different densities and dipole moments are given in Tab. 1 [10]. The initial susceptibility given by (8) can be obtained from the expression $\chi=\partial M / \partial H_{0}$ at zero external field, if the effective field is replaced by the following in (1): 


$$
H_{e}=\frac{1+p}{1-p}\left(H_{0}+\frac{4 \pi}{3} \rho m L\left(\alpha_{0}\right)\right)
$$

Equation (9) ensures that the initial part of the magnetization curve starts with the required slope. Although at higher external field even more overestimates the simulations like any of the former theories.

Table 1. The probability of the chaining formation at three different dipole moments and wide range of density.

\begin{tabular}{|c|c|c|c|}
\cline { 2 - 4 } \multicolumn{1}{c|}{} & \multicolumn{3}{c|}{$\mathbf{p}$} \\
\hline $\boldsymbol{\rho}^{*}$ & $\mathbf{m}^{*} \mathbf{1}$ & $\mathbf{m}^{*}=\mathbf{2}^{\mathbf{0 . 5}}$ & $\mathbf{m}^{*}=\mathbf{3}^{\mathbf{0 . 5}}$ \\
\hline $\mathbf{0 . 1}$ & 0.000260 & 0.000263 & 0.000580 \\
\hline $\mathbf{0 . 2}$ & 0.000426 & 0.000429 & 0.000943 \\
\hline $\mathbf{0 . 3}$ & 0.000615 & 0.000617 & 0.00794 \\
\hline $\mathbf{0 . 4}$ & 0.000852 & 0.00292 & 0.0250 \\
\hline $\mathbf{0 . 5}$ & 0.00117 & 0.0110 & 0.0521 \\
\hline $\mathbf{0 . 6}$ & 0.00164 & 0.0253 & 0.0953 \\
\hline $\mathbf{0 . 7}$ & 0.00241 & 0.0515 & 0.170 \\
\hline $\mathbf{0 . 8}$ & 0.0135 & 0.107 & 0.320 \\
\hline $\mathbf{0 . 8 5}$ & 0.0269 & 0.164 & 0.471 \\
\hline $\mathbf{0 . 9}$ & 0.0539 & 0.273 & 0.649 \\
\hline
\end{tabular}

\section{THEORY}

\subsection{The approach of effective field at high external field}

As mentioned above, except the Langevin theory the former approaches overestimates the magnetization at high external magnetic field. For describing the magnetization in all range of external field a new, correct expression has to be found at high external field, then the formulas which have concerned to the low and high external field has to be put together. The general failure of the previous theories were just that those lumped together the magnetization in the all range. At low external field with appropriate parameters the chains are formed, but do not stand in the direction of this field. Nevertheless at high external field the direction of chains is parallel to this external field. The overestimating of magnetization may also come from that the former approaches the particles regard as $\mathrm{Lm}$ sized, fixed, external field parallel particles, instead of $m$ sized, swinging ones. In the following a new modification of the effective field will be introduced, which decreases the magnetization at high external field.

It is noticeable from Fig. 1, that decreasing the $4 \pi / 3$ factor by an appropriate value in (1) the simulation data could be reproduced at high external field. The question is the rate of this reduction. At low density and dipole moment there is no need to reduce it at all, and higher density and dipole moment as shown on Fig. 1, should be reduced in a different value. For this, consider Fig. 3, where the values of effective field of Weiss theory (3) are plotted in negative external field as well to two different dipole moments and four 
different densities. Interestingly that for negative external fields belong to positive effective field, which is presumably not a stable equilibrium state.

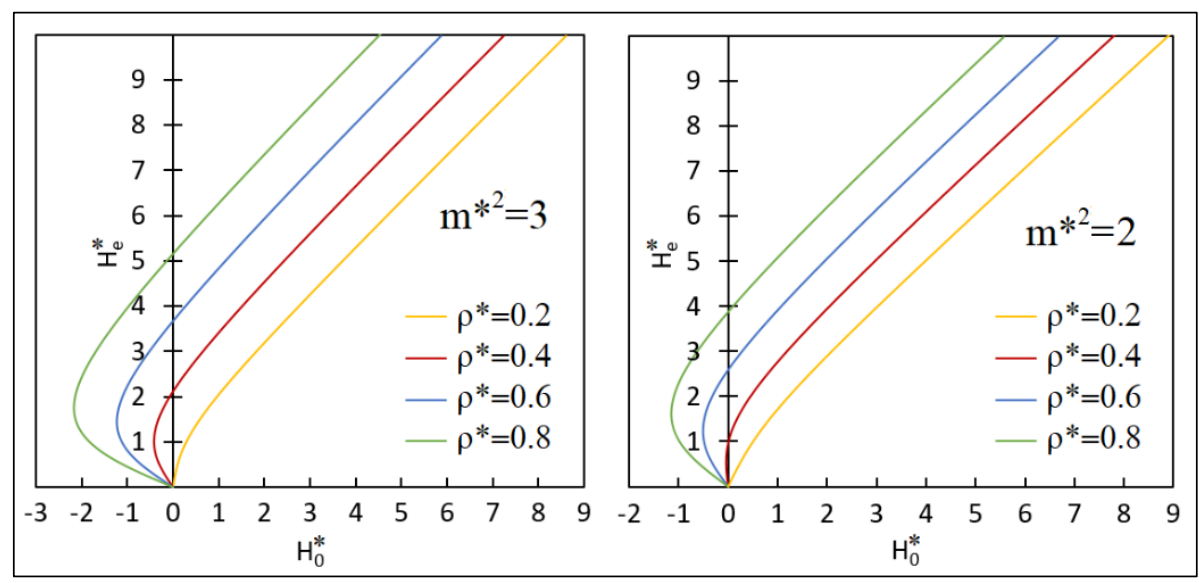

Figure 3. The values of effective magnetic field according to (3) in function of external magnetic field including the negative range at different densities and dipole moments.

The Weiss theory is applicable to approximate the simulation data at high external field if the curves are offset along the ordinate axis to negative direction on Fig. 3. Fig. 4 shows the rates of the offsets at $m^{* 2}=3$ dipole moment and three different densities: for each $H_{0}$ values must have belong to at most one $H_{e}$ value. Let's sign by $H_{0}^{\prime}$ and $H_{e}^{\prime}$ the abscissa and ordinate of the point on the curve where its slope is infinite.

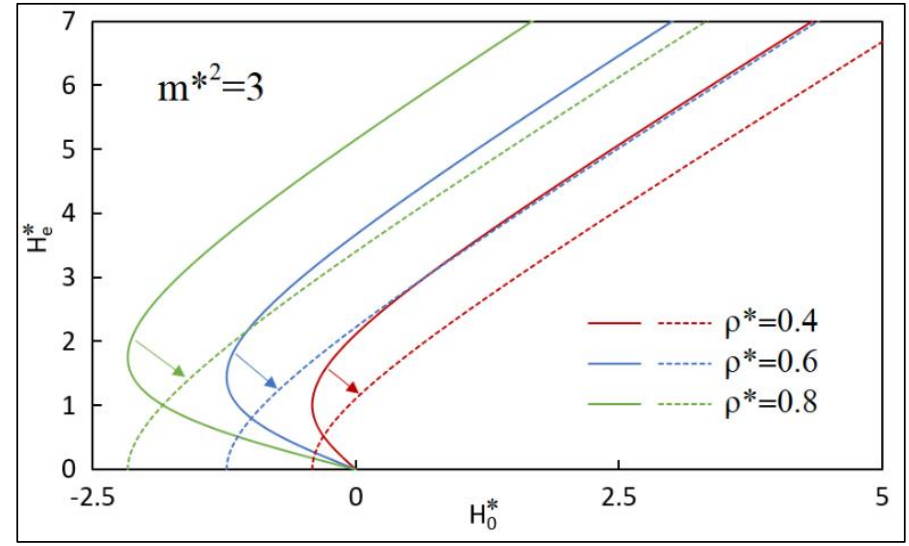

Figure 4. Examples for the extents of the offset. The dotted lines are well aproximated the simulation values of effective field 
The statement is that the rate of the mentioned offset is $H_{e}^{\prime}$, where $H_{e}^{\prime}$ is a function of $m, \rho$, and $T$. Thus (4) changes to the following expression at high external field:

$$
H_{e}=H_{0}+\operatorname{d\rho mL}\left(\alpha_{0}\right)
$$

The relation between $H_{e}^{\prime}$ and $d$ constant is:

$$
d=\frac{4 \pi}{3}-\frac{H_{e}^{\prime}}{\rho m}
$$

The value of $H_{e}^{\prime}$ can be determined from the condition that derivative of the original function at $H_{0}^{\prime}$ becomes infinite, or by other words only at $H_{0}^{\prime}$ in the range $H_{0}<0$, the function $H_{e}\left(H_{0}\right)$ has only one value. In (3) the $H_{e}$ effective field is the sum of $H_{0}$ external and $H_{p}$ additional field:

$$
H_{e}=H_{0}+H_{p}
$$

where

$$
H_{p}=\frac{4 \pi}{3} \rho m L\left(\alpha_{0}+\alpha_{p}\right)
$$

So the $H_{0}=H_{0}^{\prime}$ value is to be determined, where (13) has exactly one solution in the negative range. Let the left side be the function $f=f\left(H_{p}\right)$, while the right side is the function $g=g\left(H_{p}\right)$ and by plotting both in function of $H_{p}$, the solution of (13) is illustrativable (Fig. 5).

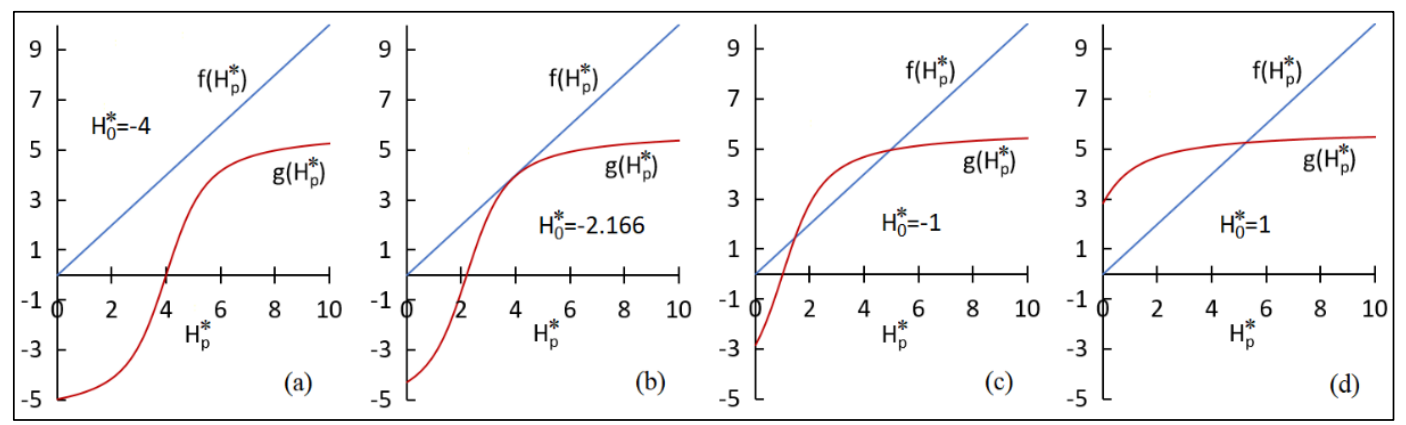

Figure 5. The graphical solution of (13). Changing the external magnetic field the number of solutions are also changing. 5.a: there is no solution if $\mathrm{H}_{0}<\mathrm{H}_{0}^{\prime} ; 5 . b$ : there is only one solution, and the two curves are tangent to each other if $\mathrm{H}_{0}=\mathrm{H}_{0}^{\prime} ; 5 . c$ : there are two solutions if $\mathrm{H}_{0}^{\prime}<\mathrm{H}_{0} \leq 0$; 5 .d: there is only one solution and the two curves intersect each other if $\mathrm{H}_{0}>0$. 
Since at $H_{0}^{\prime}$ the functions $f$ and $g$ are tangential therefore additional constraint can be determined for their differentials:

$$
\frac{\partial f\left(H_{p}\right)}{\partial H_{p}}=\left.\frac{\partial g\left(H_{p}\right)}{\partial H_{p}}\right|_{H_{p}=H_{p}^{\prime}}=1
$$

Performing the second operation, we get

$$
\frac{4 \pi}{3} \rho m^{2}\left(\alpha_{e}^{\prime-2}-\sinh ^{-2}\left(\alpha_{e}^{\prime}\right)\right)=1
$$

This equation has to be solved numerically for $H_{e}^{\prime}$. Then substituting $H_{e}^{\prime}$ into (13) can also be obtained $H_{0}^{\prime}$ and $H_{p}^{\prime}$. Furthermore, the $d$ constant and the $H_{e}$ function can be written according (11) and (10), and ultimately from (1) the magnetization can be approached in case of high external magnetic field.

Table 2. The values of $H_{0}^{\prime *}$ and $H_{e}^{\prime *}$ calculated numerically from (15) at three different dipole moments and wide range of density.

\begin{tabular}{|c|c|c|c|c|c|c|}
\cline { 2 - 7 } \multicolumn{1}{c|}{} & \multicolumn{2}{c|}{$\mathbf{m}^{*}=\mathbf{1}$} & \multicolumn{2}{c|}{$\mathbf{m}^{*}=\mathbf{2}^{\mathbf{0 . 5}}$} & \multicolumn{2}{c|}{$\mathbf{m}^{*}=\mathbf{3}^{\mathbf{0 . 5}}$} \\
\hline $\boldsymbol{\rho}^{*}$ & $\boldsymbol{H}_{\mathbf{0}}^{*}$ & $\boldsymbol{H}_{\boldsymbol{e}}^{*}$ & $\boldsymbol{H}_{\mathbf{0}}^{\prime *}$ & $\boldsymbol{H}_{\boldsymbol{e}}^{* *}$ & $\boldsymbol{H}_{\mathbf{0}}^{\prime *}$ & $\boldsymbol{H}_{\boldsymbol{e}}^{*}$ \\
\hline $\mathbf{0 . 1}$ & 0 & 0 & 0 & 0 & 0 & 0 \\
\hline $\mathbf{0 . 2}$ & 0 & 0 & 0 & 0 & 0 & 0 \\
\hline $\mathbf{0 . 3}$ & 0 & 0 & 0 & 0 & -0.1052 & 0.6387 \\
\hline $\mathbf{0 . 4}$ & 0 & 0 & -0.0410 & 0.5347 & -0.4141 & 1.0055 \\
\hline $\mathbf{0 . 5}$ & 0 & 0 & -0.2401 & 0.9613 & -0.7989 & 1.2507 \\
\hline $\mathbf{0 . 6}$ & 0 & 0 & -0.5072 & 1.2315 & -1.2276 & 1.4438 \\
\hline $\mathbf{0 . 7}$ & 0 & 0 & -0.8141 & 1.4409 & -1.6860 & 1.6069 \\
\hline $\mathbf{0 . 8}$ & -0.0579 & 0.7562 & -1.1485 & 1.6159 & -2.1664 & 1.7501 \\
\hline $\mathbf{0 . 8 5}$ & -0.1150 & 0.9495 & -1.3238 & 1.6944 & -2.4132 & 1.8162 \\
\hline $\mathbf{0 . 9}$ & -0.1823 & 1.1062 & -1.5034 & 1.7683 & -2.6638 & 1.8792 \\
\hline
\end{tabular}




\subsection{The synthesis of theories of the effective field}

In section 1.2 and 2.1 the effective field has been written at zero and high external field by (9) and (10). Now the synthesis of these expressions is needed. For better comprehension let's introduce the functions $A=A\left(H_{0}\right)$ and $B=B\left(H_{0}\right)$. The effective field can be written:

$$
H_{e}=A\left(H_{0}+B \rho m L\left(\alpha_{0}\right)\right)
$$

where:

$$
A \rightarrow \frac{1+p}{1-p} \quad \text { and } \quad B \rightarrow \frac{4 \pi}{3}, \quad \text { if } \quad H_{0} \rightarrow 0
$$

and

$$
A \rightarrow 1 \text { and } B \rightarrow d, \quad \text { if } \quad H_{0} \rightarrow \infty
$$

For these transitions the well-nown tanh function proved to be suitable with argument $m H_{0} / k_{B} T$. The transition to function $A$ is implemented by the following expression:

$$
A\left(H_{0}\right)=\left(1-\tanh \left(\alpha_{0}\right)\right)\left(\frac{1+p}{1-p}-1\right)+1
$$

After simple transformation can be written as

$$
A\left(H_{0}\right)=\frac{1+p-2 p \tanh \left(\alpha_{0}\right)}{1-p}
$$

The applicable transition to function $B$ is:

$$
B\left(H_{0}\right)=\left(1-\tanh \left(\alpha_{0}\right)\right)\left(\frac{4 \pi}{3}-d\right)+d
$$

Similar to previous transitions function $B$ is

$$
B\left(H_{0}\right)=\frac{4 \pi}{3}-\frac{H_{e}^{\prime}}{\rho m} \tanh \left(\alpha_{0}\right)
$$

In (16) function $B$ is multiplied by the Langevin magnetization, which contains the coth function, since

$$
L(x)=\operatorname{coth}(x)-\frac{1}{x}
$$


Due to the relationship between tanh and coth function the second part of (16) is:

$$
\operatorname{B\rho mL}\left(\alpha_{0}\right)=\left(\frac{4 \pi}{3}-\frac{H_{e}^{\prime}}{\rho m} \tanh \left(\alpha_{0}\right)\right) \rho m L\left(\alpha_{0}\right)=\frac{4 \pi}{3} \rho m L\left(\alpha_{0}\right)+H_{e}^{\prime}\left(\frac{\tanh \left(\alpha_{0}\right)}{\alpha_{0}}-1\right)
$$

Substituting (19) and (23) in (16) the final formula of the effective magnetic field is obtained:

$$
H_{e}=\frac{1+p-2 p \tanh \left(\alpha_{0}\right)}{1-p}\left[H_{0}+\frac{4 \pi}{3} \rho m L\left(\alpha_{0}\right)+H_{e}^{\prime}\left(\frac{\tanh \left(\alpha_{0}\right)}{\alpha_{0}}-1\right)\right]
$$

\section{RESULTS AND DISCUSSION}

Monte Carlo simulations have been performed for dipolar hard sphere fluids to determine the magnetization. The simulation values of the effective field are calculated from (1). We applied canonical NVT ensemble, Boltzmann sampling, periodic boundary conditions and minimum-image convention [11]. In order to take into account the long-ranged character of the dipolar interaction the reaction-field method was used. After 100.000 equilibration cycles, 1-3 million production cycles were used. The number of particles was $N=512$. The magnetization was obtained by summarizing the components of the dipoles in the direction of external magnetic field:

$$
M=\frac{1}{V} \sum_{i=1}^{N} \boldsymbol{m}_{i} \frac{\boldsymbol{H}_{0}}{\left|\boldsymbol{H}_{0}\right|}
$$

Our results on Fig. 6 have been presented at 2-2 different values of densities and dipole moments. The effective field based on (24) and the magnetization as well are depicted in function of external magnetic field. In contrast with Fig. 1 and 2 the simulation and theoretical results show good agreement. In case of former theories the most striking differences were experienced at the ,elbow”, around $H_{0}^{*} \approx 1$. By applying tanh function in (18) and (20) this problem was handled. 


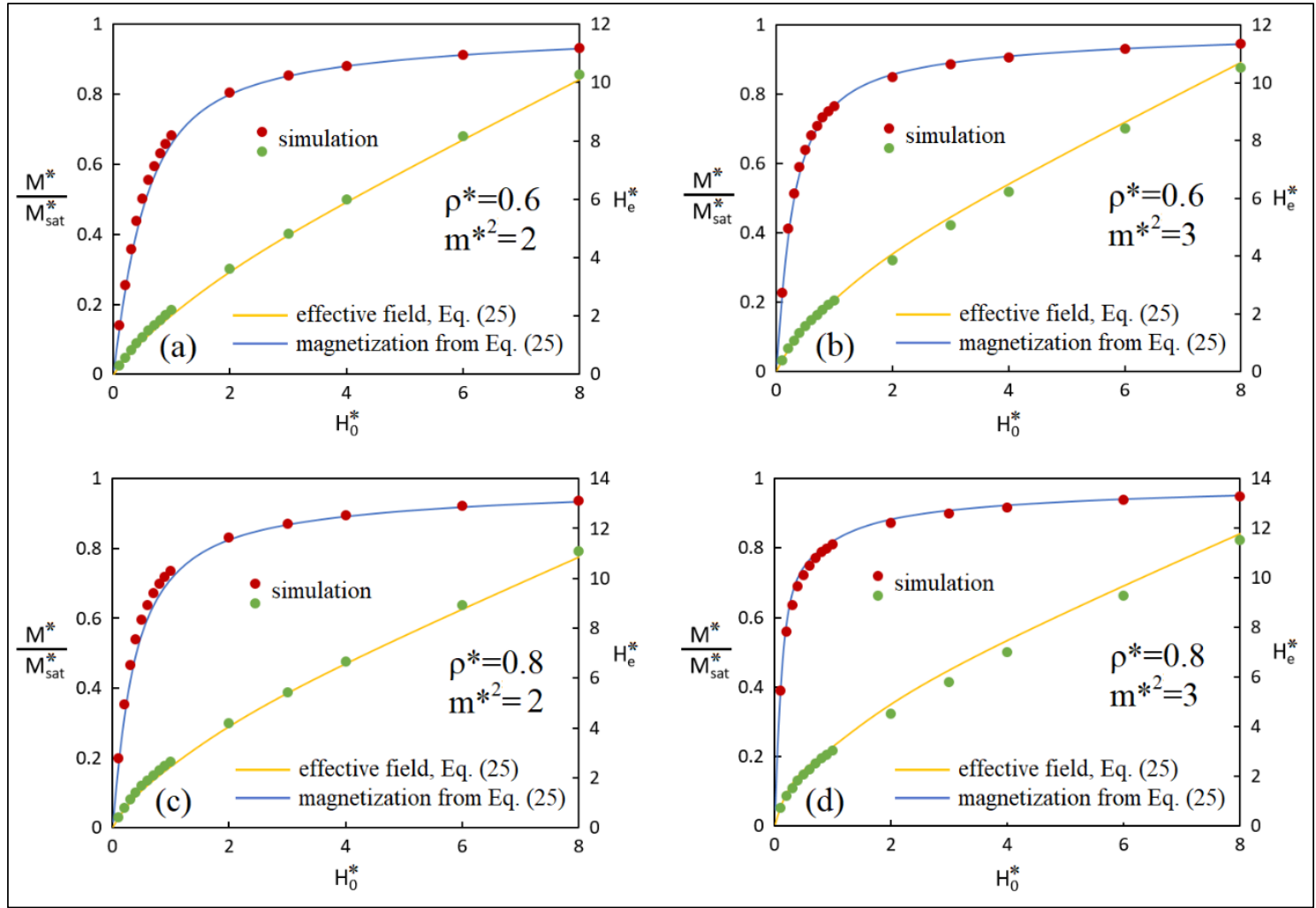

Figure 6. The values of magnetization curve (left axis) and effective magnetic field (right axis) in function of external magnetic field according to the present theory (24) comparing to the simulations.

According to Tab. 2 and (24) at low density and high external field the present theory is reproduced the results of former theories, because the values of $H_{e}^{\prime}$ are zero. Similarly according to Tab. 1 and (24) at low density and low external field the present theory is reproduced the results of former theories, because the values of $p$ converge to zero. It can be said that our theory works well up to $\rho^{*}=0.85$. Our study has shown perfectly the delusion that the magnetism can be handled theoretically jointly at low and high external field. At low external field the chains are not parallal to this external field, but they can be handled as macroparticles [10]. While at high external field the chains are more or less parallel to the external field, thus the magnetization is determined by the orientation of individual particles forming these chains. 


\section{CONCLUSION}

In this paper a new magnetization formula has been introduced for dense aggregated dipolar hard sphere fluids. At low external magnetic field the expression of susceptibility [10] has been used, while at high external field the reduction of effective magnetic field has been led to good results. The merging of these two theories was done with the help of tanh function. It is importand to emphasize that the final formula (24) does not contain empirical elements. The calculation of $p$ chaining probability variable was done numerically [10], but not by fitting. The reduction of effective field was justified mathematically, because as the Fig. 3 shows in certain cases the $H_{e}\left(H_{0}\right)$ function is divalent, furthermore the particles in the chains are not fixed, but are swinging. The calculation of this reduction was also numerical (15). As shown in Fig. 6 our theory works well at high density and dipole moment as well.

\section{REFERENCES}

[1] Langevin, P.: Sur la théorie du magnétisme, Journal de Physique Théorique et Appliquée, 1905 4, 678693

[2] Weiss, P.: L'hypothése du champ moléculaire et la propriété ferromagnétique, Journal de Physique Théorique et Appliquée, 1907 6, 661-690

[3] Pshenichnikov, A. F.; Mekhonoshin, V. V.: Equilibrium magnetization and microstructure of the system of superparamagnetic interacting particles: numerical simulation, Journal of Magnetism and Magnetic Materials, 2000 213(3), 357-369

[4] Ivanov, A. O.; Kuznetsova, O. B.: Magnetic properties of dense ferrofluids: An influence of interparticle correlations, Physical Review E, 2001 64, 041405

[5] Tani, A.; Henderson, D.; Barker, J. A.; Hecht, C. E.: Application of perturbation theory to the calculation of the dielectric constant of a dipolar hard sphere fluid, Molecular Physics, 1983 48(4), 863-869

[6] Szalai, I.; Nagy, S.; Dietrich, S.: Linear and nonlinear magnetic properties of ferrofluids, Physical Review E, 2015 92, 042314

[7] Huke, B.; Lücke, M.: Magnetization of ferrofluids with dipolar interactions: A Born-Mayer expansion, Physical Review E, 2000 62, 6875

[8] Wertheim, M. S.: Exact solution of the mean spherical model for fluids of hard spheres with permanent electric dipole moments, The Journal of Chemical Physics, 1971 55, 4291

[9] Szalai, I.; Dietrich, S.: Magnetization and susceptibility of ferrofluids, Journal of Physics: Condensed Matter, 2008 20, 204122

[10] Nagy, S.: The initial magnetic susceptibility of dense aggregated dipolar fluids, Hungarian Journal of Industry and Chemistry, Manuscript submitted for publication

[11] Allen, M. P.; Tildesley D. J.: Computer Simulation of Liquids (Clarendon Press, Oxford) 1987 